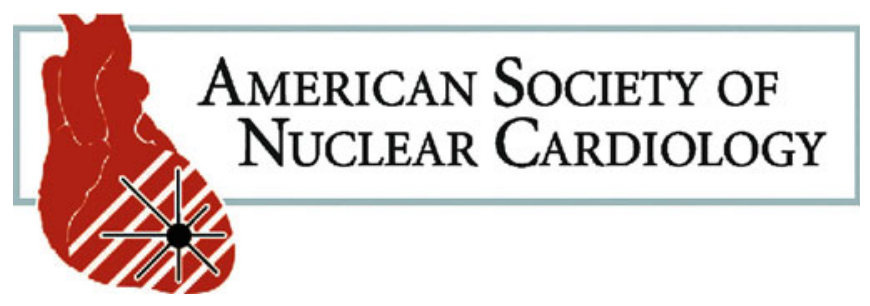

\section{CME INSTRUCTIONS: GATED SPECT IN ASSESSMENT OF REGIONAL AND GLOBAL LEFT VENTRICULAR FUNCTION: AN UPDATE}

\section{STATEMENT OF NEED}

The following educational gaps have been identified to demonstrate need for this journal continuing educational activity:

- Gated myocardial perfusion SPECT application provides enhanced diagnostic and prognostic data that may significantly improve clinical assessment and management of patients undergoing evaluation for known or suspected CAD.

- Recent developments in the gated SPECT hardware and software present a need for updated educational programs.

- Utilization of the latest gated SPECT algorithms, automated analysis applications and phase analysis is important for current clinical practice.

\section{TARGET AUDIENCE}

This activity is targeted at imaging professionals and is intended to provide the latest information on clinical practice and cutting-edge scientific advances in nuclear cardiology and cardiac imaging.

\section{OVERALL GOAL}

The overall goal of this Journal CME activity is to improve physician competence in applying evidencebased research to their individual practice situation.

\section{OBJECTIVES}

After reading and reflecting upon this article in the Journal of Nuclear Cardiology, the learner should be able to:

J Nucl Cardiol 2013;20:1116-7.

$1071-3581 / \$ 34.00$

Copyright (c) 2013 American Society of Nuclear Cardiology.

doi:10.1007/s12350-013-9811-2
- Summarize the state of the art for the utilization of gated SPECT applications in clinical practice

- Describe an incremental diagnostic and prognostic value of gated SPECT data in management of patients with known and suspected CAD.

\section{ACCREDITATION AND CONTINUING EDUCATION CREDIT}

Physicians: The American Society of Nuclear Cardiology is accredited by the Accreditation Council for Continuing Medical Education to provide continuing medical education for physicians. The American Society of Nuclear Cardiology designates this Journal-based CME activity for a maximum of 1 AMA PRA Category 1 Credits $^{\mathrm{TM}}$. Physicians should only claim credit commensurate with the extent of their participation in the activity.

Technologists: The American Society of Nuclear Cardiology is a recognized provider of continuing education credit for technologists. ASNC's Continuing Education (ACE) credit is accepted by both NMTCB and ARRT. This Journal-based activity has been approved for a maximum of 1 ACE credits for Technologists.

\section{PRINCIPAL FACULTY AND THEIR CREDENTIALS}

Editor in Chief: George A. Beller, MD, FASNC, Professor of Internal Medicine, University of Virginia;

Primary Author: Aiden Abidov, MD, PhD, Associate Professor of Medicine and Radiology,

The University of Arizona

\section{Disclosure}

As an accredited provider of the Accreditation Council for Continuing Medical Education (ACCME), The American Society of Nuclear Cardiology (ASNC) adheres to the ACCME's 2005 Standards for Commercial Support. In compliance with these standards, it is ASNC's policy to ensure balance, independence, objectivity, and scientific merit in all of its educational activities through the disclosure of relevant financial relationships with commercial companies and resolution of conflicts of interest. The financial interest or relationships requiring disclosure are outlined in ASNC's CME Conflict of Interest Policy. All persons who influenced the content of this activity are required to 
disclose any relevant financial relationships. The American Society of Nuclear Cardiology has reviewed these disclosures and resolved or managed all identified conflicts of interest through a peer review process.

The following author who was involved in the development of this activity reported a financial relationship: Aiden Abidov, MD, PhD; Grant Support: Astellas Pharma, NIH, Sarver Heart Center, Tucson, AZ; Advisory Board: Advanced Cardiac Imaging Consortium

The following members of the JNC Editorial Staff and ASNC staff who were involved in the planning and development of this activity reported no financial relationships: George A. Beller, MD, FASNC, Wendy Passerell, and Trixie Bradfield

The following question/objective reviewer who was involved in the development of this activity reported no financial relationships: Saurabh Malhotra, MD

The following question/objective reviewer who was involved in the development of this activity reported a financial relationship: Sharmila Dorbala, MD, Research Grant/Advisory Board, Astellas Pharma US, Inc.

\section{Off Label Use}

Articles may include discussion of drugs or devices, or uses of drugs or devices, that have not been approved by the Food and Drug Administration (FDA) or have been approved by the FDA for specific uses only. The FDA has stated that it is the responsibility of the physician to determine the FDA clearance status of each drug or device he or she wishes to use in clinical practice. ASNC is committed to the free exchange of medical education. Inclusion of any discussion in this program, including discussion on off-label uses, does not imply an endorsement by ASNC of the uses, products or techniques presented.

\section{Continuing Education Term of Approval}

Release Date: November 1, 2013

Expiration Date: November 1, 2014

\section{METHOD OF PARTICIPATION}

To receive a statement of credit, participants must successfully complete the quiz and evaluation questions after reading and reflecting on the article. The participant selects the single most appropriate answer for each question. A score of $75 \%$ or higher is needed to pass the quiz. If the score is less than $75 \%$, the participant will be notified and may resubmit the quiz with modified answers up to a total of three times. Tests will be graded by ASNC staff members. The exam, registration form, and evaluation can be found immediately following the CME article.

Estimated time of completion is one hour.

Send your completed registration, post-test and evaluation by mail, fax, or e-mail to:

American Society of Nuclear Cardiology

Attn: JNC Continuing Education

4340 East-West Highway, Suite 1120

Bethesda, MD 20814-4578

Fax: (301) 215-7113

E-mail: JournalCredit@asnc.org

The exam, registration and evaluation can be found immediately following the CME article.

\section{BIBLIOGRAPHY}

Bibliographic sources are cited throughout the article and a full bibliography is provided at the end of the article to provide you with further study resources on this topic.

\section{MEDIUM OR COMBINATION OF MEDIUM USED}

This article is available in print and online format.

\section{PROCESSING FEES}

ASNC members may claim continuing education credits at no charge. Non-members will be charged $\$ 50$ per activity. Please fill out the payment area included on the evaluation form.

\section{ACKNOWLEDGEMENT OF COMMERCIAL SUPPORT}

This activity is not supported by commercial support.

\section{PRIVACY \& CONFIDENTIALITY STATEMENT}

ASNC will record learner's personal information as provided on CME evaluations to allow for issuance and tracking of CME certificates. ASNC may also track aggregate responses to questions in activities and evaluations and use these data to inform the ongoing evaluation and improvement of its CME program. No individual performance data or any other personal information collected from evaluations will be shared with third parties.

For questions regarding CME content or obtaining CME credit, please contact ASNC at 301.215.7575 or info@asnc.org. 